\title{
La « Saoudisation » de l'emploi : un défi démographique autant que socio-économique, sinon politique
}

David Rigoulet-Roze

\section{(2) OpenEdition \\ Journals}

Édition électronique

URL : https://journals.openedition.org/remi/3571

DOI : 10.4000/remi.3571

ISSN : $1777-5418$

Éditeur

Université de Poitiers

\section{Édition imprimée}

Date de publication : 30 juin 2007

Pagination : $35-48$

ISBN : 978-2-911627-44-6

ISSN : 0765-0752

Référence électronique

David Rigoulet-Roze, "La « Saoudisation » de l'emploi : un défi démographique autant que socioéconomique, sinon politique », Revue européenne des migrations internationales [En ligne], vol. $23-\mathrm{n}^{\circ} 1$ । 2007, mis en ligne le 30 juin 2010, consulté le 15 avril 2022. URL : http://journals.openedition.org/ remi/3571; DOI : https://doi.org/10.4000/remi.3571

Ce document a été généré automatiquement le 15 avril 2022

(C) Université de Poitiers 


\title{
La « Saoudisation » de l'emploi : un défi démographique autant que socio-économique, sinon politique
}

\author{
David Rigoulet-Roze
}

1 En trente ans, le nombre des étrangers vivant en Arabie Saoudite s'est considérablement accru et la population nationale de plus en plus urbanisée s'est progressivement sentie « diluée » dans la masse des « étrangers » présents sur le sol du Royaume. Cette présence d'immigrés dont le nombre a crû à un rythme supérieur à celui de la population locale est clairement perçue, aujourd'hui plus encore qu'hier, comme un problème d'ordre "stratégique ». C'est dans ce contexte sensible qu'il faut trouver l'origine de la mise oeuvre d'une politique de «saoudisation » prônée par celui qui n'était encore que le Prince héritier laquelle relève somme toute d'une antienne puisque dès le 10 août 1979, Shaykh Hishâm Nâzer, alors ministre saoudien du plan, déclarait déjà dans une interview accordée à la revue Saudi Business : «Chaque Saoudien devrait se fixer pour objectif de remplacer un travailleur étranger » (Lonchampt, 1982 : 618).

\section{Le « tabou » du poids de l'immigration}

2 L'obtention de données statistiques sur le nombre précis des immigrés a toujours relevé de la plus grande difficulté. Quand elles ne sont plus inexistantes comme par le passé, elles demeurent le plus souvent soit confidentielles, soit volontairement déformées. Les chiffres exacts sur la population saoudienne demeurent généralement difficiles à établir dans la mesure où le gouvernement a toujours été réticent à les actualiser, la tendance officieuse ayant toujours été de grossir le nombre de "nationaux» par rapport à celui des "allogènes ». Cette tentation s'explique par deux types de raisons convergentes. Cela risquerait de valider les estimations sur le poids des «nonnationaux» dans l'économie du pays, et donc de mettre en valeur la fragilité démographique intrinsèque du Royaume vis-à-vis d'autres pays de la péninsule comme 
le Yémen - presque aussi peuplé que lui avec plus de 21 millions d'habitants, mais sans la prise en compte de "non-nationaux »-, et plus largement ceux du monde arabe comme l'Égypte (avec près de 79 millions d'habitants) ou l'Irak (avec près de 27 millions d'habitants), voire ceux du monde musulman, comme l'Iran (comptant près de 69 millions d'habitants) ou la Turquie (plus de 70 millions d'habitants) ${ }^{1}$.

3 L'attitude du régime saoudien résulte donc d'une volonté de masquer une présence trop importante de travailleurs étrangers sur le sol national. Selon l'avant-dernier recensement officiel datant de 1992, l'Arabie Saoudite comptait alors près de 16,6 millions d'habitants dont 4,3 millions de travailleurs immigrés. Officiellement, fin 1992, les «nationaux » saoudiens étaient donc 12,3 millions ce qui correspondrait à environ $73 \%$ du total, mais la proportion d'étrangers dans la population active représentait alors près de $80 \%$ (Bombacci, 1998: 10). Les chiffres et estimations officiels tendent systématiquement à sous-estimer le nombre des immigrés qui gardent leur nationalité d'origine. Le fait est que ceux de 1992, qui n'ont pas été publié en totalité, demeurent fortement sujets à caution. Le nombre des immigrés avait sans doute été revu à la baisse, celui des nationaux saoudiens à la hausse (Da Lage, 1996:12). Le ministère de l'Intérieur n'a-t-il pas été contraint d'admettre, en décembre 1995, la présence de 6256000 de travailleurs étrangers en Arabie Saoudite (Bombacci, 1998: 10). Quant au ministre de l'économie et du plan, Khalid bin Muhammad al-Qusaybi, il n'a pas hésité à déclarer en mai 2004 - soit quelques mois avant un recensement programmé pour le second semestre de la même année - que le Royaume comptait en réalité 8,8 millions d'étrangers, un chiffre peut-être surévalué qui lui permettait de confirmer dans le même temps que le ministère était bien décidé à réduire cette proportion à $20 \%$ au cours de huit prochaines années ${ }^{2}$.

D'où l'intérêt majeur représenté par le tout dernier recensement effectué en septembre et octobre 2004 : le 15 novembre 2004, ses résultats montraient que la population du pays (à $90 \%$ arabe, en regroupant les nationaux saoudiens et les immigrés d'origine arabe, et à $10 \%$ d'immigrés d'origine spécifiquement afro-asiatique) se montait officiellement à 22,7 millions d'habitants dont 16,5 millions de « nationaux » $(72,9 \%)$ et 6,1 millions de travailleurs « immigrés $»^{3}$ (soit 27,1\%). Mais le poids démographique de la population arabe est supérieur à son poids économique. En effet, selon les statistiques du Ministère de l'économie et du plan actualisées en 2004, près des deux tiers $(67 \%)$ de la force des travail du royaume et, de l'aveu même du ministre du travail et des affaires sociales, le Dr. Ali al-Namlah, près du quatre cinquièmes des actifs dans le secteur privé sont des immigrés, alors que moins du tiers des Saoudiens en âge de travailler ont aujourd'hui un emploi.

\section{Le Boom pétrolier et l'appel massif à la main-d'œuvre étrangère}

5 Le "problème » constitué par les travailleurs étrangers n'est pas nouveau dans la mesure où l'Arabie saoudite les a depuis longtemps utilisés pour effectuer toutes les tâches que les Saoudiens eux-mêmes répugnaient à effectuer. Avant 1974, les Yéménites étaient de loin les plus nombreux dans l'ensemble du pays. Mais la population active étant constituée aussi d'Omanais et d'Émiratis - avant qu'ils ne commencent à bénéficier de la rente pétrolière de leur propre pays - dans la partie orientale, d'Éthiopiens - souvent des musulmans venus d'Érythrée avant 
l'indépendance de cette dernière -, de Soudanais et de Nubiens sur le littoral de la Mer Rouge. Les tâches les moins ingrates et/ou celles demandant une spécialisation technique étaient plutôt du ressort d'Égyptiens, de Libanais, de Syriens, d'Irakiens et de Palestiniens de la diaspora souvent remarquablement formés (Lonchampt, 1982 : 616). Il s'agissait donc dans ces années essentiellement d'immigrants d'origine « arabe » ou, à tout le moins, de culture "musulmane». C'est ce qui faisait dire encore, le 21 mars 2002, au Prince Na'if, ministre de l'Intérieur du Royaume et, à ce titre, en charge des problèmes d'immigration: "Les Arabes et les musulmans résidant dans notre pays ne sont pas des étrangers et nous ne faisons pas de distinctions ». Une manière de reconnaître implicitement que les choses ne vont pas toujours de soi avec tous les "étrangers" présents sur le sol saoudien (Fourmont-Daimville, 2003: 43), sauf peut-être avec les expatriés occidentaux qui font figure de privilégiés (35000 Américains, 30000 Britanniques, 5000 Français, 2500 Allemands).

6 Le déficit de main-d'œuvre, tant quantitatif que qualitatif, est apparu après 1973, année $\mathrm{du}$ «choc pétrolier» qui fut synonyme de crise économique pour l'Occident, mais d'essor d'une « économie de rente » pour les pays de l'OPEP. Le gouvernement saoudien commença à faire appel à des ressortissants des pays du sous-continent indien, Pakistanais, Indiens - aujourd'hui les plus nombreux avec environ 1,5 million de personnes -, Bengladis, Sri-Lankais, mais aussi Turcs et Philippins. Ces derniers sont employés le plus souvent comme manœuvres, mais aussi comme " gens de maison », un sort peu enviable quand il ne tourne pas au cauchemar pour les «bonnes " philippines parfois victimes d'esclavage sexuel pur et simple. Le mot à connotation péjorative, sinon raciste, de paki est d'ores et déjà passé dans le langage courant pour désigner une catégorie de personnes corvéables à merci. À Riyadh, le quartier de Ba'dia, dans le vieux centre ville, est paradoxalement une "invitation au voyage » dans le sous-continent indien (Fourmont-Daimville, $2003: 42-43$ ).

7 Cette présence d'immigrés dont le nombre a crû à un rythme supérieur à celui de la population locale - essentiellement du fait d'une croissance en glissement annuel de l'offre de travail entre 1980 et 1985 de $8 \%$ (Fourmont-Daimville, 2003: 42) - est clairement perçue, aujourd'hui plus encore qu'hier, comme un problème d'ordre stratégique. Et ce, d'autant plus qu'une partie non négligeable d'entre eux sont des immigrés clandestins entrés dans le royaume de multiples manières plus ou moins légales.

\section{La pratique controversée du sponsoring}

8 Une source d'immigration clandestine est, de fait, constituée par le hajj de La Mecque qui amène chaque année plusieurs centaines de milliers de musulmans étrangers sur les « Lieux saints ». En dépit du contrôle très strict exercé par les autorités saoudiennes - en principe le visa du hajj n'est valable que pour Djeddah et les deux « villes saintes » et sa validité dépasse rarement les trois mois -, une partie non négligeable des pèlerins parvient à rester en Arabie saoudite (Bombacci, 1998 : 10).

Il existe, certes, un moyen légal d'obtenir un emploi en Arabie saoudite, surtout pour les non-musulmans: trouver un kafil ou sponsor ("parrain» saoudien) - en l'occurrence une administration publique, une entreprise privée ou un particulier saoudien - susceptible de se porter garant du comportement du l'impétrant au regard de l'ordre public en général et de la législation du travail en particulier, laquelle exclut 
naturellement le droit de grève. Cette garantie lui permet d'obtenir le précieux iqama (" permis de séjour »).

10 Mais cette procédure du sponsoring n'a pas manqué de générer tout un trafic des visas dont les sponsors sont d'une certaine manière dépositaires, ce qui leur permet de percevoir une sorte de "commission» sur le salaire des immigrés qui n'ont de toutes façons pas la possibilité de se plaindre. La réforme du sponsoring, qui devait compléter la récente réforme du code des investissements mais qui menaçait la «rente » de nombreux privilégiés avait été maintes fois annoncée et reportée, notamment du fait de l'opposition avérée du Prince Naîf qui l'avait justifiée en ces termes : «Ce n'est pas le moment. Point à la ligne! $»^{4}$. Mais à la lecture d'une étude du ministère du travail du 28 avril 2004 qui devait montrer que $70 \%$ des visas émis par l'Arabie Saoudite étaient vendus au marché noir par des sponsors peu scrupuleux, le ministre du Travail Ghazi alQusaybi, avait affirmé sa détermination à lutter contre le trafic des visas. Cette réforme d'un système juridiquement incompatible avec les règles commerciales libérales de l'Organisation Mondiale du Commerce (OMC) pour laquelle Riyadh négociait les conditions de son adhésion depuis douze ans connut finalement un début d'application qui a rendu possible l'adhésion de l'Arabie saoudite à l'OMC le 11 décembre 2005 en tant que $149^{\text {ème }}$ membre officiel de l'organisation internationale.

11 Cela ne résolvait pas pour autant le problème des travailleurs clandestins. L'expulsion de quelque 400000 «illégaux » décidées en août-octobre 1997 avaient déjà eu comme objectif affiché de démanteler les filières de recrutement mais sans remettre en cause pour autant à l'époque la monopolisation du très lucratif « commerce » des visas entre les mains des membres de la famille royale (Bombacci, 1998: 10). Cette vague d'expulsions n'était pas la première ni la dernière. Devant l'immigration irrégulière qu'il considère, à tort ou à raison, comme entraînant des risques pour la stabilité sociale et le contrôle de l'économie, le pouvoir saoudien n'a jamais hésité à prendre des mesures draconiennes qui, sous d'autres cieux, feraient le bonheur des tenants de la "préférence nationale ». Ainsi dès 1979, 88000 étrangers en situation irrégulière sontils expulsés de force (Bombacci, $1998: 10$ ).

La question de l'immigration a parfois été instrumentalisée à des fins purement «politiques» contre des voisins jugés par trop «indociles». Pour les punir d'avoir soutenu l'Irak de Saddam Hussein après l'invasion du Koweït en août 1990 et rappeler à Sanaa la pérennité de la puissance tutélaire saoudienne sur la péninsule arabique nonobstant la réunification des deux Yémens le roi Fahd par le décret du 19 septembre 1990, obligea quelques 800000 Yéménites à quitter le pays. Pourtant ces immigrés étaient depuis longtemps présents sur le sol saoudien, et libres jusque-là de s'y installer, leur présence n'était pas liée à un permis de travail parce qu'au nom du concept d'al-umma al-'arabiyya (" nation arabe ») ils ne relevaient, théoriquement, pas de la catégorie d'« étrangers » (De Klebnikoff, $1982: 208$ ). Les Saoudiens n'ont d'ailleurs pas hésité, début 1998, à chasser encore 6000 Yéménites (Bombacci, 1998 : 10). Ils sont toujours plusieurs centaines de milliers à vivre encore aujourd'hui dans le Royaume et leur nombre devrait de nouveau avoir augmenté depuis le règlement du conflit frontalier en 2000. 


\section{La difficile mise en œuvre d'une politique de "préférence nationale »}

13 Finalement, l'immigration a toujours été considérée comme une variable d'ajustement économique et politique par le pouvoir saoudien. Le fait est que l'industrie pétrolière, le bâtiment et les services dépendent principalement de la main-d'œuvre étrangère. Même l'administration n'est pas épargnée. Les étrangers constituent un quart du corps enseignant, et une part bien plus grande encore dans le secteur public de la santé : en dépit des premiers efforts "saoudisation", fin 1995, $84 \%$ des médecins, $80 \%$ des infirmiers et $55 \%$ des pharmaciens n'étaient pas des «nationaux »; cette proportion atteignait $87 \%$ pour les généralistes, $81 \%$ pour les dentistes, $79 \%$ pour les gynécologues et obstétriciens, $71 \%$ pour les pédiatres, etc... Le rapport était encore plus déséquilibré dans le secteur privé, réservé de facto aux étrangers, qui n'ont pas le droit de bénéficier de la gratuité des soins de santé dans les hôpitaux publics (Bombacci, 1998 : 10).

14 Le $7^{\text {ème }}$ Plan quinquennal de développement (2000-2004) a voulu fixer un objectif de $51 \%$ d'immigrés au maximum dans le secteur privés. Il avait prévu que les Saoudiens, employés à plus de $80 \%$ dans la fonction publique, devraient constituer dès 2001 au moins $30 \%$ des effectifs des entreprises de plus de vingt salariés. Et ce quota était censé augmenter de $5 \%$ par an afin de parvenir au remplacement de 466600 travailleurs étrangers par des actifs saoudiens à l'horizon 2005. Le nouveau code du travail achevé en octobre 2001, dispose que les Saoudiens doivent représenter au moins $75 \%$ des employés d'une entreprise. Le 30 décembre 2001, le ministère du Travail et des Affaires sociales annonça s'être fixé comme objectif pour l'année 2002, une « saoudisation » de $25 \%$ minimum dans le secteur privé (Fourmont-Daimville, $2003: 87$ ). Le 9 juillet 2002, le ministre saoudien du Travail et des Affaires sociales, le Dr. Ali bin Ibrahim Al-Nimla, publia un décret prévoyant le remplacement de la main-d'œuvre étrangère par des Saoudiens dans pas moins de 22 secteurs d'emploi.

La «saoudisation" du commerce de l'or et des postes de chauffeurs de taxis avait été annoncée à grand renfort de publicité dès octobre-novembre 2002 (Ménoret, 2003 : 167). Une loi a d'ailleurs été votée en ce sens en janvier 2003, proscrivant l'emploi d'étrangers dans la bijouterie ou parmi les chauffeurs de taxi (essentiellement Pakistanais, Afghans ou Philippins, $10 \%$ seulement étant saoudiens). Cette réduction devait être effective dans les six mois, délai porté immédiatement à deux ans et qui n’a pu qu'être prolongé. Il y a loin de la règle à l'application.

16 La «saoudisation » du commerce de l'or a dû être dans un premier temps repoussée sine die après les nombreuses protestations des employeurs. Il a fallu attendre le 21 février 2004 pour la rendre effective : au moins $50 \%$ des commerces d'or encore détenus par des étrangers (notamment des Yéménites) ont été fermés autoritairement. Par contre celle des chauffeurs de taxis a dû être largement aménagée en raison du coût excessif que l'emploi massif de «nationaux » aux prétentions salariales excessives ferait peser sur le budget des compagnies privées de taxis. On voit mal en effet comment des Saoudiens pourraient accepter les conditions de travail de ce type. La plupart des chauffeurs de taxi, originaires du sous-continent indien travaillent quatorze heures par jour, six jours par semaine, avec un salaire dépassant rarement 1000 riyals par mois (280 euros), autant dire un salaire de misère pour tout bon Saoudien habitué à l'opulence ${ }^{6}$. 


\section{Les réticences des Saoudiens pour « se mettre au travail »}

Depuis le 29 octobre 2002, toutes les demandes de visa et de permis de travail pour les travailleurs étrangers doivent passer par les bureaux du ministère du Travail et des Affaires sociales, afin de permettre à l'État de contrôler plus efficacement l'immigration (Ménoret, 2003 : 167). Le 2 mai 2004, interdiction désormais était faite aux entreprises de moins de 10 salariés de recruter des travailleurs immigrés. Une étude menée sur 75 entreprises et rendue publique le 6 mai 2004 montrait que le niveau de «saoudisation » des travailleurs du secteur privé était certes passé de $18 \%$ en moyenne en 2000 à $23 \%$ en 2004. Mais ce chiffre demeurait très en-deça des objectifs fixés par le gouvernement (de l'ordre de $38 \%$ prévu pour 2004). Le 31 mai, le ministre du Travail, Ghazi al-Qusaybi, déclarait hésiter à appliquer les "grands moyens" aux entreprises non encore "saoudisées ». Et le 6 juin suivant, le ministre du Travail, Ghazi al-Qusaybi, accordait finalement aux entreprises de moins de 10 salariés un délai de trois mois avant de mettre en œuvre la mesure de «saoudisation » totale publiée le 2 mai précédent.

Pourquoi de telles précautions? L'une des difficultés majeures à opérer une "saoudisation» réelle de l'économie tient à plusieurs facteurs qui se recoupent. En premier lieu, la jeunesse saoudienne paraît effectivement peu encline à se mettre au travail. Comme le rapporte Stéphane Marchand : «Un sondage qui circule sous le manteau montre que $21 \%$ des Saoudiens employés dans le secteur privé ne rapportent rien, ce qui s'appelle rien, à leur employeur. Même la clientèle saoudienne ne se prive pas de critiquer ses compatriotes vendeurs pour la mauvaise grâce qu'ils mettent à servir dans les magasins. (...) Chaque saoudien se rêve en chef et répugne à occuper autre chose qu'un poste de "manager". Il en est rarement capable » (Marchand, 2003, 299).

Les anecdotes concernant les Saoudiens «au travail» sont légion: entre ceux qui refusent de porter la blouse réglementaire des ouvriers par peur de subir des moqueries ou d'être arrêtés par les patrouilles de police à la recherche des clandestins ; ou ceux qui refusent la désignation d'ouvrier pour pouvoir dire à leur femme qu'ils sont techniciens; voire ceux qui ne comprennent pas pourquoi ils ne sont pas directeurs mais se trouvent obligés de côtoyer Népalais, Bengladis et autres Indiens.

Il faut dire que pendant la période correspondant à une l'ayyam al tafrah ou 'asr al-tafra («les jours de l'abondance»), l'insouciance saoudienne prévalait. Les diplômés devenaient fonctionnaires à un niveau supérieur ou entraient dans les entreprises du secteur public. Les «journées" commençaient à 10 heures pour finir vers 14 heures. D'autres pouvaient servir de prête-noms à une compagnie étrangère avec des dividendes substantiels. Les familles et surtout l'État assuraient plus que le nécessaire. Chaque étudiant touchait environ 1500 francs par mois (Gresh, $2000: 17$ ). Mais ces temps sont révolus. Toute création d'emploi dans le secteur public a été bloquée. Le chômage qui n'existait pratiquement pas il y a une dizaine d'années toucherait aujourd'hui $30 \%$ de la population, bien au-delà du taux de 8,1\% affiché en septembre 2002 par les statistiques officielles (Ménoret, 2003 : 166), alors même qu'il était déjà largement estimé à $12 \%$ dès 1999. A titre d'exemple, en 2002, plus de 1000 personnes se sont présentées aux 10 postes proposés par l'école militaire de Riyadh, provoquant une mêlée au cours de laquelle plusieurs candidats furent blessés ${ }^{7}$. 


\section{Les carences d'un système éducatif inadapté}

21 d'avoir consacré le plus clair de son temps à la lecture du Coran. L'enseignement religieux occupe entre cinq et neuf heures par semaine. Plus de $30 \%$ du temps d'étude dans le primaire est exclusivement consacré à l'approfondissement de la doctrine wahhâbite. Au collège, un cursus obligatoire de quatre ans comprend «culture islamique ", «Hadiths", «exégèse du Coran », "jurisprudence islamique » et «monothéisme islamique» (Marchand, $2003: 210$ ). Outre le fait que $40 \%$ des 200000 jeunes qui arrivent chaque année sur le marché du travail - qui n'en absorbe qu'un tiers (Ménoret, 2003: 166) - ont quitté le système scolaire sans diplôme (Ménoret, $2003: 226)^{8}$, les deux tiers des « diplômés » sortent des instituts islamiques du Royaume et, faute d'emplois, vont le plus souvent grossir les rangs des mouttawa'yn («police religieuse »). Aujourd'hui seulement 8,5\% des diplômés de l'université le sont dans des filières techniques et ne représentent que $2 \%$ des entrants sur le marché du travail (Ménoret, 2003 : 227). Ainsi, entre 1995 et 1999, quelque 114000 étudiants (dont plus de la moitié de femmes) ${ }^{9}$ sont bien sortis de l'université avec un diplôme. Mais sur ce total, à peine 10000 étaient des ingénieurs, et 16000 seulement des experts en marketing et en informatique quand 48000 avaient obtenu un diplôme d'études islamiques lequel ne présente évidemment strictement aucun intérêt pour le secteur privé (Marchand, 2003 : 285). Sur cinq années universitaires, un étudiant saoudien doit généralement valider (avec une note de 60/100) quatre unités de religion (Ummah, société islamique, économie islamique, système politique en islam). Chaque année, le système éducatif saoudien "fabrique» donc des dizaines de milliers de futurs chômeurs dont les frustrations accrues constituent une « bombe à retardement ».

\section{La croissance démographique saoudienne, une " arme » devenue une « bombe à retardement »}

La population de l'Arabie Saoudite a plus que doublé de 1980 à 1995, passant de 9 millions à 19 millions d'habitants. Le taux de croissance a, certes, diminué dans le même temps passant de 5,2 \% à 3,7 \%, mais il demeure tout de même, en 2004, l'un des plus élevés au monde avec $2,2 \%{ }^{10}$. Cela signifie que si ce taux se maintient la population ne sera à nouveau multiplié par deux, dans vingt ans, pour atteindre alors quelque 40 millions d'habitants en 2025. D'autres experts estiment au contraire ces chiffres comme excessifs et évaluent la population saoudienne de 2025 à 34 millions d'habitants dont 27 millions de «nationaux » (Courbage, $2001: 35$ ). Pour tenter d'avoir une vision objective de la situation, le 18 septembre 2001, la direction des statistiques du ministère du Plan a débuté la mise en œuvre de la première phase des préparatifs d'une campagne globale de recensement démographique dans les 13 provinces que compte le Royaume, programmée pour le second semestre 2004. Le 14 juin 2002, un rapport intérimaire du ministère du Plan révélait que la population saoudienne était d'ores et déjà estimée, fin 2001, à 20,8 millions de personnes, soit tout de même une augmentation de $4,8 \%$ en glissement annuel. Les «nationaux » étaient alors 15,6 millions et les "étrangers » 5,2 millions. Ledit rapport prévoyait que la population saoudienne pourrait s'élever

Revue européenne des migrations internationales, vol. 23 - $n^{\circ} 1$ | 2007 
seulement à 29,7 millions d'ici une vingtaine d'années, loin des estimations alarmistes précitées (Fourmont-Daimville, $2003: 39$ ).

Il paraît néanmoins difficile de nier la réalité du problème constitué par cette croissance démographique continue, avec en 2004 un taux de natalité de 29,3\%o et une fécondité de 4 enfants par femme, ce qui constitue encore l'un des records mondiaux en la matière ${ }^{11}$. Comme le souligne Antoine Sfeir, (2006: 125) «cela signifie en particulier la nécessité de construire des écoles à une cadence inconnue ailleurs (plus d'une par jour) $»^{12}$.

24 Avec $38 \%$ de la population ayant moins de quinze ans et un taux de chômage officiellement de $22 \%$ mais en réalité plutôt proche de $30 \%$, on comprend les affres du régime saoudien face à ce défi démographique. Le 3 octobre 2004, le ministère du Travail, l'Organisation générale de l'enseignement technique et le Fond de développement de la main-d'œuvre lançaient une vaste campagne visant à créer 100000 emplois en trois ans. Parallèlement, ce qui souligne combien les deux variables sont liées dans l'esprit des décideurs saoudiens, le ministre du Travail, Ghazi alQusaybi, annonçait de son côté un plan de réduction de la main-d'œuvre étrangère de quelque 100000 immigrés par an.

Le problème de la croissance démographique a été longtemps sous-estimé, pour des raisons tenant à la fois à l'islam en général, et à la doctrine wahhâbite en particulier, celle-ci ne pouvant tout simplement pas envisager l'idée d'un contrôle des naissances. Mais il y a aussi des raisons géopolitiques à cette sous-estimation, qui font de la démographie une "arme » face aux autres États plus ou moins peuplés de la péninsule. Le royaume saoudien a, en effet, toujours eu la hantise d'apparaître comme un " nain démographique " aux yeux de ses rivaux régionaux. Ce n'est plus tout à fait le cas aujourd'hui puisque l'Arabie saoudite est en quelque sorte devenue une puissance démographique représentant même $70 \%$ du CCEAG (Conseil de coopération des États Arabes du Golfe) plus connu sous acronyme de CCG (Conseil de Coopération du Golfe) ${ }^{13}$.

\section{Un pays confronté au risque d'un « 68 islamiste »}

Le problème démographique est aujourd'hui toujours aussi préoccupant mais pour des raisons rigoureusement inverses à celles d'il $\mathrm{y}$ a vingt ans dans la mesure où la structure de la pyramide des âges annonce nécessairement des jours difficiles pour le régime qui ne sait que faire d'une population dont $70 \%$ a moins de trente ans, $65 \%$ moins de 24 ans et $39 \%$ moins de 15 ans $^{14}$. De plus, avec la baisse de la rente pétrolière des années quatre-vingt-dix, le PIB par tête a considérablement baissé - de moitié en vingt ans pour devenir inférieur à celui de la Hongrie - à environ 10500 dollars en 2002 et 8690 dollars en 2003, alors qu'il se montait à 28600 dollars en 1981, soit à peu près l'équivalent de celui des États-Unis à l'époque. De plus, selon le département américain de l'énergie, compte tenu de l'inflation, le revenu par tête serait en réalité passé de 24000 dollars à seulement 2600 dollars, contre 36000 aujourd'hui pour les Etats-Unis ${ }^{15}$.

27 A l'occasion de l'interview précitée, accordée le 11 mai 2002, au rédacteur en chef du journal Al-Sharq Al Awsat, celui qui n'était encore que le Prince héritier, tout en prenant la mesure du problème, semblait, pour des considérations de politique intérieure, imputer les difficultés induites par le taux de croissance démographique moins à des mécanismes endogènes qu'à des causes conjoncturelles externes: "Depuis les années 
quatre-vingt-dix, lorsque le Royaume a dû faire face à des événements internes et externes difficiles, nous réfléchissons aux mesures susceptibles d'être adoptées pour faire face à la croissance démographique et à une source de revenus unique dépendant de fluctuations externes qu'il est impossible de contrôler [i.e. les cours du baril de brut]. Tous les règlements et les codes annoncés témoignent tout simplement de notre réalisme à nous adapter à des conditions qui n'étaient pas prévues ou qui ont été plutôt imposées de l'extérieur et qui n'entraient pas dans nos calculs. Notre seule option est de les traiter par des moyens et des méthodes pratiques sans tarder et sans tergiverser. Il est possible de tout envisager, excepté les questions qui touchent à notre chariah [i.e. l'établissement d'une politique de "planning familial" qui heurterait l'establishment wahhâbite]. C'est une question que nous refusons et sur laquelle nous n'accepterons aucun compromis ». La quadrature du cercle en quelque sorte. Et pourtant, cette croissance démographique effrénée constitue probablement un des défis majeurs que le régime saoudien ait aujourd'hui à relever s'il veut échapper à des difficultés qui pourraient devenir insurmontables.

Mai Yamani, chercheuse au Royal Institute of International Affairs de Londres et chargée de la première enquête de terrain consacrée aux jeunes d'Arabie saoudite ${ }^{16}$, insiste sur la crise d'identité affectant la jeunesse saoudienne : «Leurs grands-parents, résume-t-elle, ont vu le royaume se construire et le pétrole surgir. Leurs parents ont été les enfants gâtés du pétrole, et la monarchie était alors légitimée par la redistribution des richesses. Eux se sentent vulnérables. Ils n'ont ni les certitudes de leurs grands-parents, ni la sécurité économique de leurs parents. Leur seule référence est l'islam $»^{17}$.

\section{Les perspectives inespérées ouvertes par le renouveau du boom pétrolier}

29 Aujourd'hui, la hausse importante du prix du baril redonne une bouffée d'oxygène au régime saoudien. Il lui revient de la mettre à profit pour les générations à venir. Ce qui semble être la politique du nouveau roi Abdallah, contrairement à ce qui avait prévalu auparavant. Dans les années soixante-dix, les pétrodollars avaient été « recyclés » dans des dépenses somptuaires et l'achat massif d'armement aussi coûteux qu'inutiles, ainsi que des placements plus ou moins hasardeux. L'inflation avait fait ensuite son travail de dévaluation systématique de la rente pétrolière. Lorsque le prix du brut était finalement retombé, le gouvernement saoudien avait vu son budget asséché et il n'avait pas été en mesure de faire face aux demandes croissantes de sa population. Fort de cette amère expérience, Abdallah n'entend pas rejouer la même partition. Tout d'abord, on constate qu'une partie non négligeable des pétrodollars sert à rembourser les dettes contractées. En cinq ans, entre le début de l'an 2000 et 2005, la dette publique saoudienne a ainsi été divisée par deux, passant de $119 \%$ du PIB en 1999 à 97 \% en 2002 pour descendre à $41 \%$ en 2005. Mais la véritable nouveauté réside peut-être dans le fait qu'une partie importante des revenus pétroliers - passés de 30 milliards de dollars en 1998 à 80 en 2000, 86 en 2003 et 154 en $2005^{18}$ - est réinvestie localement non seulement dans le secteur pétrolier mais aussi dans le développement d'une économie à côté du pétrole. La croissance a ainsi été de 6,7 \% dans le secteur privé et les réserves de change de la banque centrale ont même triplé par rapport à 2002 pour se monter à 133 milliards de dollars en 2005. Priorité a été donnée, dans le budget 2006 aux dépenses sociales et à l'enseignement afin d'améliorer le secteur peu performant de l'éducation. Lors de la présentation du budget 2005 faite le 8 décembre 2004, les sommes allouées 
aux secteurs de l'éducation et de la formation se trouvaient en augmentation de $9 \%$ par rapport à 2004 (soit $25 \%$ des dépenses budgétaires).

Pour la première fois depuis 25 ans, le salaire des fonctionnaires a été augmenté de $15 \%$. Grâce aux énormes liquidités provenant du pétrole, le Royaume prévoit des investissements de 500 milliards de dollars au cours des quinze prochaines années qui devraient profondément transformer le pays si les variables géopolitiques extérieures n'affectent pas la pérennité du régime.

\section{BIBLIOGRAPHIE}

BOMBACCI Nicolas (1998) Préférence nationale à la saoudienne, Le Monde Diplomatique, octobre, p. 10.

CHAMPENOIS Lucien SOULIE Jean-Louis (1982) Le Royaume d'Arabie saoudite, in Paul Bonnenfant Éd., La péninsule arabique d'aujourd'hui. 2. Études par pays, vol. 2, pp. 565-602.

CIA (2005) World Factbook 2005 https://www.cia.gov/cia/publications/factbook/geos/sa.html COURBAGE Youssef (2001) L'Arabie saoudite : une démographie en changement, Monde Arabe Maghreb-Machrek, $n^{\circ}$ 174, octobre-décembre, Paris, La Documentation française, pp. 28-37.

COURBAGE Youssef (1999) Nouveaux horizons démographiques en Méditerranée, Paris, INED-PUF, 200 p.

DA LAGE Olivier (16 juillet 2004) Le « cauchemar » des immigrés dans le royaume, texte sur site RFI (http://www.rfi.fr/actufr/articles/055/article 29316.asp).

DA LAGE Olivier (1996) Géopolitique de l'Arabie Saoudite, Bruxelles, Éditions Complexe, 143 p. FOURMONT-DAIMVILLE Guillaume (2004) Géopolitique de l'Arabie Saoudite, Paris, Ellipses, 171 p. GRESH Alain (2000) Petite brise de mondialisation sur la société saoudienne, Le Monde diplomatique, avril, pp. 16-17.

HUMAN RIGHTS WATCH (2004) Bad Dreams: Exploitation and Abuse of Migrant Workers in Saudi Arabia, 135 p.

KLEBNIKOFF Serge de (1982) Les travailleurs immigrés de la péninsule, in Paul Bonnenfant Éd., La péninsule arabique d'aujourd'hui, vol. 1, pp. 191-218.

LONCHAMPT Jacques (1982) La planification en Arabie saoudite, in Paul Bonnenfant Éd., La péninsule arabique d'aujourd'hui, vol. 2. Etudes par pays, pp. 603-622.

MÉNORET Pascal (2005) Chronologie de l'Arabie saoudite - 2004, in Chroniques yéménites, (http:// cy.revues.org/document192.html)

MÉNORET Pascal (2003) L'énigme saoudienne. Les Saoudiens et le monde, 1744-2003, Paris, La Découverte, $260 \mathrm{p}$.

VERSION ORIGINALE (1993) La Péninsule Arabique, Paris, n 3, 267 p. 
RIGOULET-ROZE David (2006) L'oblitération de la femme saoudienne, Les Cahiers de l'Orient, $\mathrm{n}^{\circ} 82$, pp. 99-122.

SAINT-PROT Charles EL TIBI Zeina Éds. (2004) L'Arabie saoudite à l'épreuve des temps modernes, Paris, iDlivre, Observatoire d'Etudes Géopolitiques, 203 p.

SFEIR Antoine (2006) Redistribution des richesses, Les Cahiers de l'Orient, $\mathrm{n}^{\circ}$ 82, pp. 123-127.

YAMANI Mai (2000) Changed Identities: The Challenge of the New Generation in Saudi Arabia, London, Royal Institute of International Affairs, $170 \mathrm{p}$.

YAMANI Hani (1997) To be a Saudi, London, Janus Publication Company, 157 p.

\section{NOTES}

1. CIA World Factbook 2004.

2. « Expatriates Number $8.8 \mathrm{~m}$ », Arab News, May 25, 2004.

3. À ces travailleurs « immigrés » proprement dits, s'ajoutent encore un certain nombre de leurs proches ce qui fait monter le chiffre total des ressortissants «étrangers » à quelque sept millions, soit $30 \%$ de la population totale du royaume selon le ministre du travail et des affaires sociales, le Dr. Ali al-Namlah.

4. Claude Lorieux, «La crise irakienne freine les réformes saoudiennes », Le Figaro, 19 novembre 2002, p. 2.

5. Christophe Deloire, «Le démons de l'Arabie saoudite », in Le Point, n 1597, 25 avril 2003.

6. Yves-Michel Riols, « L'Arabie saoudite, royaume sur un volcan », L'Expansion, 29 janvier 2003.

7. Neil MacFarqhuhar, «Rien ne va plus au paradis des pétrodollars », Le Courrier International, $\mathrm{n}^{\circ} 573,25$ octobre 2001.

8. Si un quart des enfants échappe encore à l'école, tout aussi grave est le fait que $40 \%$ des enfants scolarisés sortent du système scolaire sans atteindre le collège. Cela signifie avant tout que la moitié des jeunes Saoudiens entre sur le marché du travail sans aucune qualification. (Pascal Ménoret, 2003 : 226).

9. Sur le caractère inversement proportionnel du nombre des femmes dans la société saoudienne et de leur poids économique réel, voir Rigoulet-Roze, 2006.

10. Cf. CIA World factbook 2005.

11. Cf. CIA World factbook 2005.

12. Cf. Antoine Sfeir, "Redistribution des richesses ", Les Cahiers de l'Orient, deuxième trimestre 2006, n 82, pp. 123-127, p. 125.

13. Selon le Rapport du CCEAG de juillet 2002 (Fourmont-Daimville, $2003: 38$ ).

14. Cf. CIA World factbook 2005.

15. Cf. Dominique Gallois, « Le blason terni de l'or noir », « Horizons », Le Monde, 3-4 mars 2002, p.

14.

16. Cf. Mai Yamani, Changed Identities: The Challenge of the New Generation in Saudi Arabia, 2000.

17. Cf. Dominique Lagarde, "Arabie Saoudite: les jeunes en panne de repères ", L'Express, 15 novembre 2001.

18. Cf. « Arabie saoudite : données générales » (http://www.elysee.fr/elysee/elysee.fr/francais/ actualites/deplacements_a_1_etranger/2006/mars/fiches/arabie_saoudite/1_arabie_saouditedonnees_generales.42197.html). 


\section{RÉSUMÉS}

L'obtention de données statistiques fiables sur la population immigrée en Arabie Saoudite a toujours relevé du défi, l'attitude du gouvernement saoudien ayant toujours été de grossir le proportion des «nationaux» au détriment de celle des "allogènes» dont le nombre est généralement perçu comme une menace pour la stabilité du pays. C'est dans ce contexte sensible qu'il faut d'ailleurs trouver l'origine d'une politique de «saoudisation " par Abdallah avant même son accession formelle au trône en août 2005. Mais cette "saoudisation » souhaitée rencontre nombre d'obstacles difficultés à surmonter: la jeunesse saoudienne paraît peu encline à «se mettre au travail» et souffre d'un déficit de formation et de compétences du fait de l'inadaptation $\mathrm{du}$ système éducatif. L'économie saoudienne apparaît, pour l'heure, dans l'impossibilité d'absorber la masse des jeunes qui arrivent chaque année sur le marché du travail. La croissance démographique très élevée, longtemps envisagée comme une "arme » par le pouvoir saoudien qui a toujours eu la hantise d'apparaître comme un "nain démographique » aux yeux de ses rivaux régionaux, apparaît aujourd'hui comme une "bombe à retardement ". Depuis peu, la nouvelle hausse importante du prix du baril redonne une bouffée d'oxygène au régime saoudien. Il lui revient de la mettre à profit pour les générations à venir en effectuant les réformes nécessaires.

A "Saoudization" of Employment: a Demographic, Socio-economical and Political Challenge. Getting reliable statistics on the immigrants in Saudi Arabia has always represented a challenge, as the constant attitude of the Saudi government has always been to inflate the percentage of "nationals" at the expense of "non natives", whose number is usually perceived as a threat to the stability of the country. The origin of the policy of "Saoudization" led by Abdullah even before he formally accessed the throne in 2005 has to be found in that sensitive context. But the hoped for "Saoudization" meets a number of difficult to overcome obstacles: Young Saudians do not seem very much inclined to "get down to work", and suffer a lack of training and abilities, because of the maladjustment of the education system. For the time being, The Saudi economy appears unable to absorb the bulk of young people who access the labour market each year. A very high demographic growth, long considered a "weapon" for the Saudi power, which has always been haunted by the fear to be considered a "demographic dwarf" by its rivals in the region, appears to day as a "time bomb". Very recently, a new and important increase in the price of the oil barrel has been giving the Saudi regime a puff of fresh air. It now has to make good use of it for future generations by carrying out the necessary reforms.

La "Saudizacion" del empleo : un desafío tan demográfico como socioeconómico y político. La obtención de datos estadísticos fiables sobre la población inmigrante en Arabia Saudita es siempre un desafió. La actitud del gobierno saudita a continuamente consistido en incrementar la proporción de "nacionales" con detrimento a la de "alógenos" cuyo numero se percibe generalmente como una amenaza para la estabilidad del país. En el marco de este contexto sensible nace la política de "saudización" de Abdallah antes incluso de que éste llegue al trono en agosto de 2005. Mas esta "saudización" se topa con obstáculos de difícil sobrepaso: la juventud saudí parece poco inclinada al trabajo y sufre un déficit de formación y competencias como consecuencia de la inadaptación del sistema educativo. La economía saudí se encuentra, por el momento, ante la imposibilidad de absorber la masa de jóvenes que se incorpora cada año al mercado de trabajo. El crecimiento demográfico, muy elevado, y que durante mucho tiempo ha sido interpretado como un "arma" para el poder saudí que temía dar la imagen de "enano demográfico" ante sus rivales regionales, se ha convertido hoy en día en una "bomba de relojería". Desde hace poco, el nuevo crecimiento importante del precio del barril vuelve a dar un 
balón de oxígeno al régimen saudí. Le corresponde a éste aprovechar esta situación para realizar las reformas necesarias con vistas a las generaciones futuras.

INDEX

Mots-clés : croissance démographique, éducation, formation, marché du travail, politique économique

Index géographique : Arabie Saoudite

\section{AUTEUR}

\section{DAVID RIGOULET-ROZE}

Chercheur à l'IFAS (Institut Français d'Analyse Stratégique), Collège Interarmées de la Défense, IFAS@strato-analyse.org. 